\title{
Patient-prosthesis mismatch after transapical aortic valve implantation: Incidence and impact on survival
}

\author{
Marian Kukucka, MD, ${ }^{\mathrm{a}}$ Miralem Pasic, MD, PhD, ${ }^{\mathrm{a}}$ Stephan Dreysse, MD, ${ }^{\mathrm{a}}$ Alexander Mladenow, MD, ${ }^{\mathrm{a}}$ \\ Helmut Habazettl, MD, PhD, ${ }^{\mathrm{a}, \mathrm{b}}$ Roland Hetzer, MD, PhD, ${ }^{\mathrm{a}}$ and Axel Unbehaun, $\mathrm{MD}^{\mathrm{a}}$
}

\begin{abstract}
Objectives: Transcatheter aortic valve implantation (TAVI) has become an important therapeutic option for high-risk patients with severe aortic valve stenosis. Patient-prosthesis mismatch (P-PM) is an important determinant of morbidity and mortality after open aortic valve replacement. The objective of our study was to evaluate P-PM incidence and its impact on survival in a large cohort of patients treated with TAVI.
\end{abstract}

\begin{abstract}
Methods and Results: We retrospectively analyzed transesophageal echocardiographic data of 278 consecutive patients (Society of Thoracic Surgeons score $18.5 \pm 15.3$, age $80 \pm 8$ years) who underwent transapical TAVI with Edwards Sapien valves between April 2008 and March 2011. Effective orifice area was calculated using the continuity equation and indexed with body surface area (iEOA). P-PM was stratified as severe (iEOA $<0.65$ $\mathrm{cm}^{2} / \mathrm{cm}^{2}$ ) and moderate (iEOA, $0.65-0.85 \mathrm{~cm}^{2} / \mathrm{m}^{2}$ ). Midterm survival (up to 30 months) was analyzed by Kaplan-Meier curves and log-rank tests. There was no P-PM in $181(65.1 \%)$ patients; moderate P-PM was found in $76(27.3 \%)$ patients and severe P-PM in $21(7.6 \%)$. Thirty-day survival was $96.0 \%, 97.3 \%$, and $90.5 \%$. The 3-month survival was $91 \%, 90 \%$, and $66 \%$, respectively $(P=.0013)$. Combination of severe P-PM with peak pressure gradients greater than $10 \mathrm{~mm} \mathrm{Hg}$ further reduced the 3-month survival to $48 \%$. Additionally, mean survival time in patients with an ejection fraction less than $50 \%$ was significantly shorter than in patients with an ejection fraction greater than $50 \%(20.8 \pm 1.5$ vs $24.1 \pm 0.8$ months; $P=.027)$.
\end{abstract}

Conclusions: P-PM is found in patients undergoing transapical TAVI. Severe mismatch is accompanied by high early mortality, especially when combined with increased pressure gradients. (J Thorac Cardiovasc Surg 2013;145:391-7)

Aortic valve stenosis is an important cause of cardiac morbidity and mortality in elderly patients. ${ }^{1}$ Replacement of the aortic valve with a prosthesis is the only treatment having an impact on mortality in symptomatic patients. Despite technical developments to optimize valve prostheses, the rheologic properties of prostheses are not comparable with those of native human valves, and aortic stenosis is traded for prosthesis hemodynamics. This patient-prosthesis mismatch (P-PM) was described by Rahimtoola ${ }^{2}$ in 1978 as consisting of the effective prosthetic valve area less than that of a normal human valve. Nowadays, P-PM is assumed when the effective orifice area (EOA), determined with the continuity equation, of a normally functioning prosthesis is too small in relation to the patient's body size and results in increased transprosthetic pressure gradients during hemodynamic states of higher cardiac output. A clinically used

\footnotetext{
From Deutsches Herzzentrum Berlin, ${ }^{\mathrm{a}}$ and the Institute of Physiology, ${ }^{\mathrm{b}}$ Charité Campus Benjamin Franklin, Berlin, Germany.

Disclosures: Drs Pasic, Unbehaun, and Dreysse have been proctors to Edwards Lifesciences since July 2009. The other authors have nothing to disclose with regard to commercial support.

Received for publication Oct 21, 2011; revisions received Dec 22, 2011; accepted for publication Jan 16, 2012; available ahead of print Feb 13, 2012.

Address for reprints: Pasic Miralem, MD, PhD, Deutsches Herzzentrum Berlin, Augustenburger Platz 1, 13353 Berlin, Germany (E-mail: pasic@dhzb.de). $0022-5223 / \$ 36.00$

Copyright (c) 2013 by The American Association for Thoracic Surgery doi:10.1016/j.jtcvs.2012.01.043
}

parameter for the characterization and detection of P-PM is EOA indexed with body surface area (iEOA). Patients with severe P-PM have an iEOA less than $0.65 \mathrm{~cm}^{2} / \mathrm{m}^{2}$ and those with moderate P-PM an iEOA of between 0.65 and $0.85 \mathrm{~cm}^{2} / \mathrm{m}^{2}{ }^{3}$ The incidence of severe P-PM after surgical aortic valve replacement (AVR) is $2 \%$ to $11 \%$ and of moderate P-PM $20 \%$ to $70 \%$ and is related to adverse clinical outcome. ${ }^{4}$

Transcathether aortic valve implantation has become a therapy option in high-risk patients with severe aortic stenosis. ${ }^{5-7}$ The procedure reduces the surgical risk and enables easier and faster postoperative recovery. Periprocedural clinical decision making during transcatheter aortic valve implantation (TAVI) is based on various imaging methods including computed tomography, echocardiography, and angiography. ${ }^{8}$ Among these imaging techniques, transesophageal echocardiography (TEE) seems to be superior for preprocedural geometric measurements, risk assessment, rapid diagnosis of intraprocedural complications, and assessment of functional hemodynamic characteristics after valve implantation. ${ }^{9}$ In our institution exact postprocedural echocardiographic assessment of left ventricular (LV) function, valve function, geometric orifice area (GOA), and EOA is included in the standard TEE evaluation immediately after TAVI. ${ }^{6,8,10}$

The aim of this study was to evaluate the incidence of P-PM in a large cohort of patients treated with transapical 


Abbreviations and Acronyms
$\begin{aligned} \text { AVR } & =\text { aortic valve replacement } \\ \text { EF } & =\text { ejection fraction } \\ \text { EOA } & =\text { effective orifice area } \\ \text { iEOA } & \text { effective orifice area indexed with } \\ & \text { body surface area } \\ \text { GOA } & \text { geometric orifice area } \\ \text { LV } & =\text { left ventricle (ventricular) } \\ \text { LVEDV } & =\text { left ventricular end-diastolic volume } \\ \text { LVEF } & =\text { left ventricular ejection fraction } \\ \text { LVESV } & =\text { left ventricular end-systolic volume } \\ \text { LVOT } & =\text { left ventricular outflow tract } \\ \text { P-PM } & =\text { patient-prosthesis mismatch } \\ \text { TAVI } & =\text { transcatheter aortic valve implantation } \\ \text { TEE } & =\text { transesophageal echocardiography }\end{aligned}$

TAVI with a balloon-expandable transcatheter valve and to analyze the impact of P-PM and periprocedurally acquired echocardiographic parameters on early and midterm survival.

\section{METHODS \\ Patients}

Between April 2008 and March 2011, we enrolled in the study 278 patients treated with TAVI using Edwards Sapien valves (Edwards Lifesciences, Irvine, Calif). Written informed consent was obtained from all patients. The study was approved by our institutional review committee. Our initial results, technical considerations, institutional policies, and early outcomes have been published elsewhere. ${ }^{6,8,10}$ Follow-up was between 1 and 30 months with a total of 3916 patient-months and was complete in all patients. Except for the few hospital deaths, survival data were obtained from the public register of residence.

\section{TAVI Procedure}

The valve was implanted through a mini left anterior thoracotomy via the transapical route using balloon-expandable transcatheter stent-prosthetic xenograft valves 23 or $26 \mathrm{~mm}$ in diameter with their delivering system (both Edwards Sapien THV, Edwards Lifesciences). All procedures were performed by our team of cardiac surgeons, a cardiologist, and anesthesiologists in our hybrid operation room equipped with a monoplane angiography system. A perfusionist and a heart-lung machine were at stand-by in the operating room. The surgical technique as described in detail by Walther and colleagues ${ }^{11}$ was used with some modifications. The most important modification was slow and gradual valve deployment (as opposed to rapid deployment by forced inflation of the balloon in the original technique) supported by simultaneous angiographic visualization. ${ }^{10}$ This visualization of the aortic root during slow valve deployment enables optimal positioning of the valve with perfect presentation of the relationships among the prosthetic valve, the aortic valve annulus, aortic cusps, and coronary arteries. The valve was always implanted during a short phase of rapid ventricular pacing. Additionally, the main steps of the procedure were monitored by intraprocedural TEE, namely, visualization and correct positioning of the guide wire across the native aortic valve via the transapical path, transapical introduction of the $14 \mathrm{Fr}$ soft sheath and then the $26 \mathrm{Fr}$ delivery system, assessment of ventricular function after rapid pacing and balloon valvuloplasty, and assessment of the implanted valve, the coronary arteries, and global and regional LV and right ventricular function immediately after implantation.

\section{Valve Size Selection}

The size of the valve used was determined according to the diameter of the native aortic valve annulus and aortic root morphology assessed by intraoperative TEE. We chose a valve size of $23 \mathrm{~mm}$ for aortic valve annuli smaller than $21 \mathrm{~mm}$ and $26 \mathrm{~mm}$ for annuli between $21 \mathrm{~mm}$ and $24 \mathrm{~mm}$. In borderline cases, the decision was made on an individual basis, taking into account additional factors such as the distance from the annulus to the coronary artery ostia, the shape of the annulus (oval vs circular), the amount of calcification in the leaflets, or aortic diameters at the level of the sinuses of Valsalva.

\section{Intraprocedural and Postprocedural TEE Measurements}

TEE was used to quantify preprocedural and postprocedural aortic valve geometry and hemodynamics. According to the recommendations of the American Society of Echocardiography, ${ }^{12}$ the standard TEE views for assessment of LV function and aortic valve morphology and hemodynamics were acquired and digitally stored. All examinations were performed with the same type of echocardiography equipment (Vivid I; GE Vingmed Ultrasound, Horten, Norway) during steady state periods after induction of anesthesia, after valve implantation, and at the end of the procedure before termination of anesthesia.

\section{Analyses of the Echocardiographic Data}

A retrospective quantitative analysis of periprocedural echocardiographic data was performed by an experienced echocardiographer who was blinded to outcome parameters using the Echopac work station (Echopac; GE Vingmed Ultrasound). Measurements were averaged from 3 to 5 beats.

\section{Function}

For quantification of LV systolic function, we applied the Simpson biplane method and calculated end-diastolic and end-systolic volumes and the resulting ejection fraction.

\section{Pressure Gradients}

To quantify peak and mean pressure gradients across the aortic valve, we traced the outer edge of the Doppler signal curve across the aortic valve. The maximal pressure gradient is calculated from the maximum velocity (Vmax) using a simplified Bernoulli equation: $\Delta \mathrm{Pmax}=4 \times \mathrm{Vmax}^{2}$. The mean gradient is calculated by averaging the instantaneous gradients during the whole ejection period.

\section{EOA}

To quantify the EOA, we used the continuity equation based on the concept that the stroke volume in the left ventricular outflow tract (LVOT) and across the aortic valve is equal. Stroke volume in Doppler echocardiography is calculated as cross-sectional area times the average flow velocity during the ejection period (velocity time integral, VTI). The crosssectional area (CSA) in the LVOT is calculated using the LVOT diameter. We traced the dense velocity curve in the LVOT and the outer edge of the velocity curve across the aortic valve (AV). The resulting values were included in the continuity equation to obtain the EOA: EOA = VTI LVOT $\times$ CSA LVOT/VTI AV.

\section{GOA}

GOA was quantified by planimetry using the edge of the calcified or implanted valve from the aortic valve short-axis view in midsystole. 


\section{P.PM}

The EOA was indexed by body surface area (iEOA) to consider the cardiac output requirements of the patients. Moderate P-PM was considered to be present if iEOA was from $0.65 \mathrm{~cm}^{2} / \mathrm{m}^{2}$ to $0.85 \mathrm{~cm}^{2} / \mathrm{m}^{2}$, and severe P-PM was defined as iEOA smaller than $0.65 \mathrm{~cm}^{2} / \mathrm{m}^{2}$.

\section{Statistics}

Preprocedural and postprocedural echocardiographic data are presented as mean \pm standard deviation. Changes were analyzed by paired $t$ tests. Patient survival was analyzed using Kaplan-Meier curves and differences between groups were assessed by log-rank tests with Holm-Sidak all pairwise comparisons for P-PM. Patients were assigned to groups according to clinically established cutoff values of echocardiographic parameters (iEOA, LV ejection fraction [LVEF], heart rate) or according to optimal cutoff values derived from receiver operating characteristics curves (LV enddiastolic volume [LVEDV], LV end-systolic volume [LVESV]). Multivariate survival analyses were performed for 3-month and 30-month survivals using a Cox regression proportional hazards model. Independent predictors for severe P-PM were identified by multivariate logistic regression. Differences among groups with no, moderate, and severe P-PM were assessed by the Kruskal-Wallis test followed by the Dunn post hoc multiple comparisons or by $\chi^{2}$ tests.

\section{RESULTS}

\section{Patient Cohort}

Of 301 patients who underwent TAVI, 7 received a second valve during the same procedure (to treat significant paraprosthetic leakage or transvalvular regurgitation after implantation of the first Edwards Sapien valve) and 16 underwent so-called "valve-in-valve" transapical TAVI ${ }^{13}$ owing to degeneration of a previously surgically implanted biological aortic valve prosthesis. These 23 patients were excluded from the study. In total, data from 278 patients were analyzed. The mean patient age was $80 \pm 8$ years, the mean logistic EuroSCORE was $38.0 \% \pm 20.3 \%$, and The Society of Thoracic Surgeons score was $18.5 \% \pm 15.3 \%$. A $23-\mathrm{mm}$ valve was implanted in 91 patients and a 26-mm valve in 187.

\section{LVEF}

LV systolic function improved immediately after TAVI (Table 1). Biplane ejection fraction (EF) increased from $53 \% \pm 17 \%$ to $59 \% \pm 15 \%(P<.00001)$. Both LVEDV and LVESV decreased, which was slightly more noticeable in LVESV (LVEDV, $-24 \%, P=.002$; LVESV, $-28 \%$, $P<.00001)$. Correlation analyses revealed a strong inverse dependence of LVESV on LVEF $(R=-0.77 ; P<.00001)$.

\section{Transaortic Pressure Gradients}

Periprocedural changes in aortic valve hemodynamics as assessed by TEE are also given in Table 1. Peak and mean transaortic pressure gradients decreased from $63 \pm 23$ and $38 \pm 14 \mathrm{~mm} \mathrm{Hg}$ to $10.5 \pm 5.8$ and $5.4 \pm 3.0 \mathrm{~mm} \mathrm{Hg}$, respectively.

\section{EOA}

After TAVI, EOA increased from a preimplant value of $0.51 \pm 0.17$ to a postimplant value of $1.76 \pm 0.44 \mathrm{~cm}^{2}$.
TABLE 1. Echocardiographic findings before and after TAVI

\begin{tabular}{lccc}
\hline \multicolumn{1}{c}{ Variable } & Pre-TAVI & Post-TAVI & P value \\
\hline EF $(\%)$ & $53 \pm 17$ & $59 \pm 15$ & $<.00001$ \\
LVEDV $(\mathrm{mL})$ & $88 \pm 113$ & $68 \pm 32$ & .002 \\
LVESV $(\mathrm{mL})$ & $41 \pm 32$ & $31 \pm 24$ & $<.00001$ \\
LVOT SV $(\mathrm{mL})$ & $49 \pm 17$ & $51 \pm 19$ & .08 \\
LVOT CO $(\mathrm{L} / \mathrm{min})$ & $2.8 \pm 1.0$ & $3.7 \pm 1.4$ & $<.00001$ \\
HR $($ beats $/ \mathrm{min})$ & $58 \pm 16$ & $74 \pm 17$ & $<.00001$ \\
LVOT Vmax $(\mathrm{m} / \mathrm{s})$ & $0.71 \pm 0.21$ & $0.98 \pm 0.28$ & $<.00001$ \\
dPmax $(\mathrm{mm} \mathrm{Hg})$ & $63 \pm 23$ & $10.5 \pm 5.8$ & $<.00001$ \\
dPmean $(\mathrm{mm} \mathrm{Hg})$ & $38 \pm 14$ & $5.4 \pm 3.0$ & $<.00001$ \\
AV annulus $(\mathrm{mm})$ & $21.8 \pm 1.6$ & $17.9 \pm 1.6$ & $<.00001$ \\
GOA $\left(\mathrm{cm}^{2}\right)$ & $0.70 \pm 2.0$ & $2.06 \pm 0.35$ & $<.00001$ \\
EOA $\left(\mathrm{cm}^{2}\right)$ & $0.51 \pm 0.17$ & $1.76 \pm 0.44$ & $<.00001$ \\
iEOA $\left(\mathrm{cm}^{2} / \mathrm{m}^{2}\right)$ & $0.28 \pm 1.0$ & $0.98 \pm 0.26$ & $<.00001$ \\
EOA $/ \mathrm{GOA}^{2}$ & $0.76 \pm 0.24$ & $0.85 \pm 0.18$ & $<.00001$ \\
\hline Ejen
\end{tabular}

Ejection fractions and left ventricular volumes were calculated by the biplane method. All data are means \pm standard deviation. $P$ values are derived from paired $t$ tests. TAVI, Transcatheter aortic valve implantation; $E F$, ejection fraction; $L V E D V$ and $L V E S V$, left ventricular end-diastolic and end-systolic volumes; LVOT SV and LVOT CO, stroke volume and cardiac output calculated from left ventricular outflow tract diameter and mean blood flow velocity; HR, heart rate; LVOT Vmax, peak LVOT blood flow velocity; dPmax, peak transvalvular pressure gradient; dPmean, mean transvalvular pressure gradient; AVannulus, aortic valve annulus diameter; $G O A$, geometric orifice area; $E O A$, effective orifice area; $i E O A$, indexed effective orifice area

EOA was only weakly related to preprocedural annulus size $(R=0.31 ; P<.00001)$ or severity of preprocedural stenosis (preprocedural EOA; $R=0.14 ; P=.023$ ).

\section{GOA}

Postprocedurally assessed GOA correlated reasonably with EOA $(R=0.57 ; P<.00001)$ and was greater than EOA with a mean ratio of EOA/GOA of $0.85 \pm 0.18$.

\section{P-PM}

Indexed EOA increased from $0.28 \pm 1.0$ to $0.98 \pm 0.28$ $\mathrm{cm}^{2} / \mathrm{m}^{2}$. There was no P-PM in $181(65.1 \%)$ patients; moderate P-PM (iEOA 0.65 to $0.85 \mathrm{~cm}^{2} / \mathrm{m}^{2}$ ) was found in 76 $(27.3 \%)$ patients, and severe P-PM (iEOA $<0.65$ $\left.\mathrm{cm}^{2} / \mathrm{m}^{2}\right)$ in $21(7.6 \%)$. Independent predictors of severe P-PM were body surface area $(P=.00002)$ and LVOT diameter $(P=.04)$. At a cutoff of $1.885 \mathrm{~m}^{2}$, body surface area predicted severe P-PM with a sensitivity of 0.71 and a specifity of 0.70 (odds ratio, 5.65; confidence intervals [CI], 2.11-15.11; $P=.0004)$.

Patient characteristics for all patients, patients with no, moderate, and severe P-PM are given in Table 2 . The only differences between groups are the significantly larger body weight, body surface area, and body mass index in patients with P-PM.

Valve and hemodynamic characteristics according to the groups are listed in Table 3. Patients with severe P-PM have significantly lower EOA, stroke volume, and significantly higher valve gradients in comparison with the groups with moderate or no P-PM. P-PM was unrelated to annulus 
TABLE 2. Preprocedural parameters of patient group as a whole and divided into subgroups taking account of postprocedural P-PM

\begin{tabular}{|c|c|c|c|c|c|}
\hline & All & No P-PM & Moderate P-PM & Severe P-PM & $P$ value \\
\hline $\mathrm{N}$ & 272 & 180 & 71 & 21 & \\
\hline Sex male & $81(30 \%)$ & $59(33 \%)$ & $17(24 \%)$ & $5(24 \%)$ & .32 \\
\hline Age (y) & $80 \pm 8$ & $80 \pm 7$ & $78 \pm 11$ & $79 \pm 6$ & .27 \\
\hline BW (kg) & $72 \pm 16$ & $69 \pm 13$ & $77 \pm 19 *$ & $89 \pm 21^{*}, \dagger$ & .0001 \\
\hline $\operatorname{BSA}\left(\mathrm{m}^{2}\right)$ & $1.81 \pm 0.21$ & $1.76 \pm 0.18$ & $1.87 \pm 0.24 *$ & $2.00 \pm 0.24^{*}$ & .0001 \\
\hline $\mathrm{BMI}\left(\mathrm{kg} \cdot \mathrm{m}^{-2}\right)$ & $26.8 \pm 5.6$ & $25.6 \pm 4.5$ & $28.3 \pm 6.3^{*}$ & $31.8 \pm 7.8^{*}$ & .0001 \\
\hline CAD & $157(58 \%)$ & $99(55 \%)$ & $45(63 \%)$ & $13(62 \%)$ & .44 \\
\hline NYHA & $3.30 \pm 0.49$ & $3.33 \pm 0.48$ & $3.25 \pm 0.49$ & $3.26 \pm 0.54$ & .52 \\
\hline Log. EuroSCORE & $37.8 \pm 20.2$ & $38.4 \pm 20.2$ & $35.8 \pm 19.2$ & $39.9 \pm 24.0$ & .69 \\
\hline STS Mortality Score & $18.5 \pm 15.2$ & $18.1 \pm 14.7$ & $18.8 \pm 15.4$ & $21.4 \pm 18.9$ & .73 \\
\hline Creatine clearance $\ddagger\left(\mathrm{mL} \cdot \min ^{-1}\right)$ & $52 \pm 24$ & $48 \pm 21$ & $60 \pm 30$ & $59 \pm 24$ & .0019 \\
\hline ProBNP $(\mathrm{ng} / \mathrm{mL})$ & $5.3 \pm 8.4$ & $5.3 \pm 9.2$ & $5.1 \pm 6.4$ & $5.7 \pm 7.4$ & .940 \\
\hline
\end{tabular}

Data are means \pm SD or numbers (percent); Differences among groups were evaluated by Kruskal-Wallis test followed by the Dunn post hoc multiple comparisons and by $\chi^{2}$ tests. $P$-PM, Patient-prosthesis mismatch; $B W$, body weight; $B S A$, body surface area; $B M I$, body mass index; $C A D$, coronary artery disease; NYHA, New York Heart Association; $S T S$, The Society of Thoracic Surgeons; $B N P$, brain natriuretic peptide. $* P<.05$ versus no P-PM. $\dagger P<.05$ versus moderate P-PM. $\ddagger$ Estimated by Cockcroft-Gault formula.

size and LVEF. In the group without P-PM, 26-mm prostheses were implanted more frequently $(72 \%)$ than in the group with severe P-PM $(52 \% ; P=.058)$.

\section{Survival}

Mean observation time was $14 \pm 10$ month. The overall 30-day mortality was $4.0 \%$ (11 patients died). Survival at $1,6,12,24$, and 30 months was $96.0 \%, 85.8 \%, 82.3 \%$, $65.7 \%$, and $61 \%$, respectively. Survival curves of patients with severe or moderate P-PM versus patients without P$\mathrm{PM}$ are presented in Figure 1. Thirty-day survival of patients without P-PM, with moderate P-PM, and with severe P-PM was $96.0 \%, 97.3 \%$, and $90.5 \%$, respectively. Three-month survival of patients without P-PM, with moderate P-PM, and with severe P-PM was $91 \%, 90 \%$, and $66 \%$, respectively $(P=.0013)$. Only severe P-PM was associated with an increased mortality within the first 3 months $(P=.0013)$.
This difference was maintained until 12 months ( $P=.039)$ but leveled off thereafter ( $P=.19$ at 30 months $)$.

The group of patients with severe P-PM $(n=21)$ was analyzed in more detail (Table 4). The only significant difference between the 14 survivors and the 7 nonsurvivors was a greater peak transvalvular pressure gradient in the nonsurvivors $(19.8 \pm 10.8$ vs $11.5 \pm 5.5 \mathrm{~mm} \mathrm{Hg} ; P=.02)$. Within the small group of patients with severe P-PM, 14 had a peak pressure gradient greater than $10 \mathrm{~mm} \mathrm{Hg}$, which was associated with a survival rate of $48 \%$ at 3 months, whereas all of the 7 patients with a peak pressure gradient less than 10 $\mathrm{mm} \mathrm{Hg}$ survived $(P=.029)$. Also, 5 of the patients with severe P-PM had an LVEF less than $50 \%$, which tended to be associated with a lower survival at 3 months than LVEF greater than $50 \%(40 \%$ vs $75 \% ; P=.051)$. Between survivors and nonsurvivors in this group, there was no difference in EuroSCORE (35.7 \pm 20.4 vs $48.4 \pm 29.7$;

TABLE 3. Valve characteristic and hemodynamic data of patient group as a whole and divided into sub-groups taking account of post-procedural PPM

\begin{tabular}{|c|c|c|c|c|c|}
\hline & All & No P-PM & Moderate P-PM & Severe P-PM & $P$ value \\
\hline AV annulus (mm) & $21.8 \pm 1.6$ & $21.9 \pm 1.6$ & $21.6 \pm 1.7$ & $21.8 \pm 1.5$ & .46 \\
\hline Prosthessis $26 \mathrm{~mm}$ & $182(67 \%)$ & $129(72 \%)$ & $42(59 \%)$ & $11(52 \%)$ & .056 \\
\hline $\operatorname{EOA}\left(\mathrm{cm}^{2}\right)$ & $1.8 \pm 0.4$ & $2.0 \pm 0.4$ & $1.4 \pm 0.2 *$ & $1.2 \pm 0.1 *$ & .0001 \\
\hline $\operatorname{iEOA}\left(\mathrm{cm}^{2} \cdot \mathrm{m}^{-2}\right)$ & $0.98 \pm 0.26$ & $1.12 \pm 0.21$ & $0.75 \pm 0.06$ & $0.59 \pm 0.04$ & ND \\
\hline LVOT SV (mL) & $51 \pm 19$ & $55 \pm 19$ & $44 \pm 15^{*}$ & $38 \pm 11^{*}$ & .0001 \\
\hline LVOT CO $\left(\mathrm{L} \cdot \min ^{-1}\right)$ & $3.7 \pm 1.4$ & $4.0 \pm 1.5$ & $3.3 \pm 1.2 *$ & $2.9 \pm 0.8^{*}$ & .0001 \\
\hline HR (beats/min) & $76 \pm 17$ & $75 \pm 17$ & $76 \pm 18$ & $78 \pm 15$ & .81 \\
\hline LVEDV biplane (mL) & $69 \pm 32$ & $69 \pm 31$ & $67 \pm 35$ & $70 \pm 33$ & .45 \\
\hline $\mathrm{EF}(\%)$ & $59 \pm 15$ & $59 \pm 15$ & $59 \pm 14$ & $58 \pm 19$ & .99 \\
\hline$\Delta \mathrm{Pmax}(\mathrm{mm} \mathrm{Hg})$ & $10.5 \pm 5.8$ & $9.5 \pm 5.0$ & $11.8 \pm 6.1 *$ & $14.3 \pm 8.4^{*}$ & .0002 \\
\hline$\Delta$ Pmean $(\mathrm{mm} \mathrm{Hg})$ & $5.4 \pm 3.0$ & $4.9 \pm 2.5$ & $6.3 \pm 3.4 *$ & $7.5 \pm 4.1^{*}$ & .0001 \\
\hline LVOT Vmax $\left(\mathrm{m} \cdot \mathrm{s}^{-1}\right)$ & $0.98 \pm 0.28$ & $0.99 \pm 0.28$ & $0.96 \pm 0.28$ & $0.98 \pm 0.27$ & .59 \\
\hline
\end{tabular}

Data are means \pm standard deviation. Differences among groups were evaluated by Kruskal-Wallis test followed by Dunn's post hoc multiple comparisons. $P$-PM, Patient-prosthesis mismatch; AV annulus, aortic valve annulus diameter; iEOA, indexed effective orifice area; $L V O T$ SV and LVOT CO, stroke volume and cardiac output calculated from left ventricular outflow tract diameter and mean blood flow velocity; $H R$, heart rate; $L V E D V$, left ventricular end-diastolic volume; $E F$, ejection fraction; $\triangle d P m a x$, change in peak transvalvular pressure gradient; $\triangle d P$ mean, change in mean transvalvular pressure gradient; LVOT Vmax, peak LVOT blood flow velocity. $* P<.05$ versus no P-PM. 


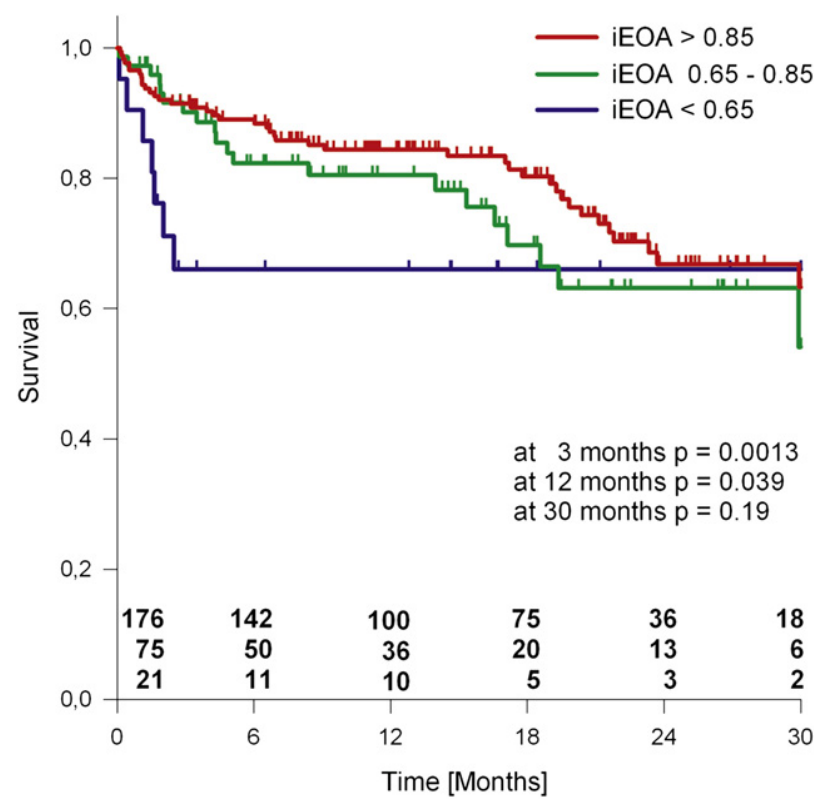

FIGURE 1. Kaplan-Meier survival curves for patients without P-PM (iEOA > 0.85), with moderate P-PM (iEOA 0.65-0.85), and with severe P-PM $(\mathrm{iEOA}<0.65)$. Censored patients are represented by vertical marks. Only severe P-PM was associated with increased early mortality within the first 3 months after TAVI. This effect was confirmed in a multivariate analysis. The difference in survivals persisted for 1 year and leveled off thereafter. $P$ values were derived from the $\log$-rank test nd all pairwise comparisons by Holm-Sidak (adjusted $P=.0014$ and .009 for severe versus no and moderate P-PM at 3 months and .037 and .13 at 12 month, respectively). P-PM, Patient-prosthesis mismatch; $i E O A$, indexed effective orifice area; TAVI, transcatheter aortic valve implantation.

$P=.26)$ or in Society of Thoracic Surgeons score (17.4 \pm 12.6 vs $29.3 \pm 26.9 ; P=.43$ ).

Additionally, postprocedural LVEF, LVESV, and heart rate show significant impact on 30-month survival. Survival was decreased in patients with an EF less than $50 \%$ than in patients with EF greater than $50 \%(78 \%, 47 \%$, and $37 \%$ vs

TABLE 4. Echocardiographic findings in patients with severe P-PM (iEOA $<0.65 \mathrm{~cm}^{2} / \mathrm{m}^{2}$ )

\begin{tabular}{lccc}
\hline \multicolumn{1}{c}{ Variable } & Survivors & Nonsurvivors & $\boldsymbol{P}$ value \\
\hline $\mathrm{N}$ & 14 & 7 & \\
EF $(\%)$ & $61 \pm 16$ & $51 \pm 23$ & .34 \\
LVEDV $(\mathrm{mL})$ & $64 \pm 26$ & $84 \pm 46$ & .39 \\
LVESV $(\mathrm{mL})$ & $26 \pm 17$ & $46 \pm 40$ & .34 \\
LVOT SV $(\mathrm{mL})$ & $35.4 \pm 9.1$ & $42.3 \pm 13.8$ & .28 \\
EOA $\left(\mathrm{cm}^{2}\right)$ & $1.16 \pm 0.08$ & $1.20 \pm 0.16$ & .68 \\
iEOA $\left(\mathrm{cm}^{2} / \mathrm{m}^{2}\right)$ & $0.59 \pm 0.04$ & $0.58 \pm 0.06$ & .47 \\
dPmax $(\mathrm{mm} \mathrm{Hg})$ & $11.5 \pm 5.5$ & $19.8 \pm 10.8$ & .02 \\
dPmean $(\mathrm{mm} \mathrm{Hg})$ & $6.3 \pm 2.6$ & $9.9 \pm 5.5$ & .07 \\
\hline
\end{tabular}

$\mathrm{EF}$ and LV volumes were calculated by the biplane method. All data are means \pm standard deviation. $P$ values are derived from unpaired $t$ tests. $P$ - $P M$, Patient-prosthesis mismatch; $i E O A$, indexed effective orifice area; $E F$, ejection fraction; $L V E D V$, left ventricular end-diastolic volume; LVESV, left ventricular end-systolic volume; $E O A$, effective orifice area; $d P \max$, peak transvalvular pressure gradient; dPmean, mean transvalvular pressure gradient.
$82 \%, 71 \%$, and $71 \%$ at, respectively, 12,24 , and 30 months; $P=.027$ ) (Figure 2). Also, patients with a LVESV greater than $35 \mathrm{~mL}$ show reduced survival compared with those with an LVESV less than $35 \mathrm{~mL}(74 \%, 46 \%$, and $37 \%$ vs $85 \%, 72 \%$, and $72 \%$ at, respectively, 12,24 , and 30 months; $P=.002$ ). Postprocedural heart rate greater than 80 beats/min had a negative impact on survival (76\%, $53 \%$, and $49 \%$ vs $86 \%, 75 \%$, and $70 \%$ at, respectively, 12,24 , and 30 months; $P=.005$ ). Postprocedural LVEDV, transvalvular peak, or the mean pressure gradients had no significant impact on survival. In the multivariate analyses, severe P-PM and heart rate were confirmed as independent predictors of 3-month survival whereas heart rate and serum creatinine concentration were associated with 30-month survival (Table 5). In contrast, LVEF and LVESV were not confirmed as independent predictors of 30-month survival.

\section{DISCUSSION}

Our study demonstrates that quantitative TEE data acquired during the intraprocedural course of TAVI are of interest also for postprocedural risk stratification and decision making. The major finding of our study is that postprocedural severe P-PM has a marked independent impact on 3-month survival in high-risk patients undergoing TAVI. This effect is even more pronounced when severe P-PM is combined with a transvalvular peak pressure gradient greater than $10 \mathrm{~mm} \mathrm{Hg}$. In addition, serum creatinine concentration and postprocedural heart rate, LVEF, and LVESV were associated with survival, but the contribution of LVEF and LVESV was not confirmed in the multivariate analysis.

Prevalence of severe P-PM in our TAVI group was 7.6\% and thus comparable with that in patients undergoing AVR or TAVI $(2 \%-13 \%) .{ }^{3,14-17}$ Blais, ${ }^{3}$ Rabus, ${ }^{17}$ and their associates identified severe P-PM (iEOA $\left.<0.65 \mathrm{~cm}^{2} / \mathrm{m}^{2}\right)$ as an independent predictor of short-term mortality among patients undergoing AVR. Concurrently, in our group severe P-PM also had a significant effect on short-term survival. However, beyond the critical period of 3 months, the prognosis of patients with severe P-PM was comparable with that of patients with iEOA greater than $0.65 \mathrm{~cm}^{2} / \mathrm{m}^{2}$. Additional analysis of patients with severe P-PM shows that the combination of severe P-PM with a peak transvalvular pressure gradient greater than $10 \mathrm{~mm} \mathrm{Hg}$ (which occurred in our 14 patients) was particularly detrimental, resulting in a 3-month survival of only $48 \%$, whereas all 7 of the patients with a lower pressure gradient survived this early period. Increased pressure gradients result in increased LV workload, which may explain their impact on mortality. Although the average peak pressure gradient in this subgroup was only moderately increased $(17.6 \pm 8.5 \mathrm{~mm} \mathrm{Hg})$, it is necessary to keep in mind that it was measured during steady state at rest and may markedly increase during even moderate physical exertion. Despite the low numbers 

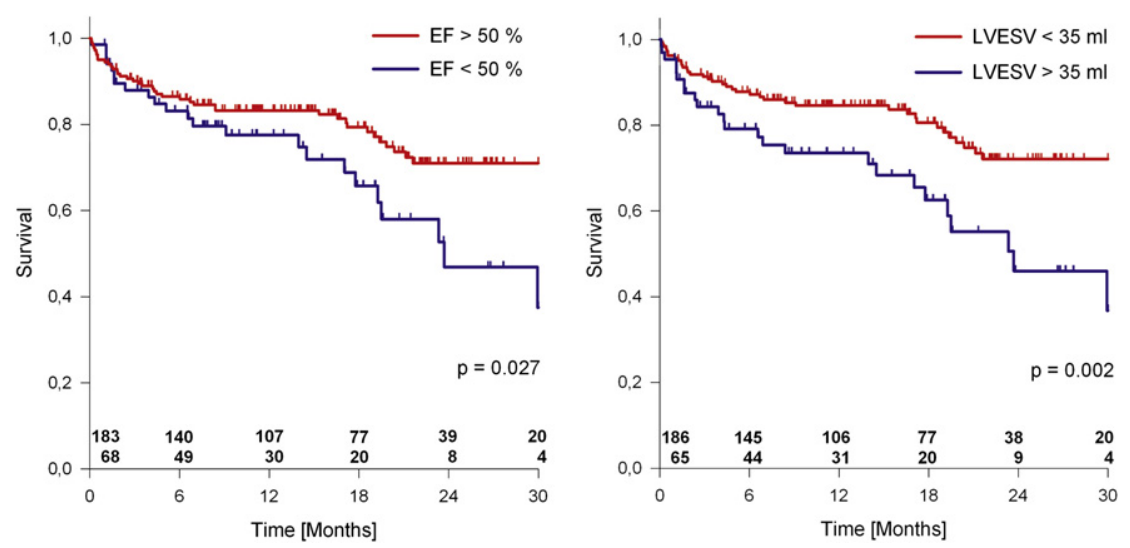

FIGURE 2. Kaplan-Meier survival curves for patients with ejection fraction $(E F)$ greater than $50 \%$ versus less than $50 \%$ (left panel) and for patients with left ventricular end-systolic volume ( $L V E S V$ ) less than $35 \mathrm{~mL}$ versus greater than $35 \mathrm{~mL}$ (right panel). Cutoff values for risk stratification were selected from receiver operating characteristics curves. Censored patients are represented by vertical marks. $P$ values were derived from log-rank tests. The association of EF and LVESV with survival was, however, not confirmed in the multivariate analysis.

of patients with severe P-PM (21 patients) or a combination of severe P-PM and change in peak pressure gradient greater than $10 \mathrm{~mm} \mathrm{Hg}$ (14 patients), these findings may have immediate clinical impact. Therefore, theoretically, an attempt to increase postprocedural iEOA might be considered (ie, by redilatation of the implanted valve or by surgical AVR). Especially for the latter decision, the risks of this intervention must be carefully weighed against the anticipated benefits. Alternatively, after recovery from the procedure, pressure gradients could be measured during stress echocardiography and further decisions based on these results. However, before an unequivocal clinical recommendation can be given, more studies on the incidence of severe P-PM with or without accompanying increased pressure gradients after TAVI and its impact on survival are needed.

Walther and colleagues ${ }^{18}$ used the expected EOA provided by valve manufacturers divided by body surface

TABLE 5. Results of multivariate Cox regression proportional hazards model

\begin{tabular}{|c|c|c|c|c|}
\hline & \multicolumn{2}{|c|}{ Three months } & \multicolumn{2}{|c|}{ Thirty months } \\
\hline & HR (CI) & $\begin{array}{c}P \\
\text { value }\end{array}$ & HR (CI) & $\begin{array}{c}P \\
\text { value }\end{array}$ \\
\hline P-PM & $4.41(1.71-11.41)$ & .002 & $1.25(0.52-2.97)$ & .62 \\
\hline $\begin{array}{l}\text { Heart rate } \\
\quad \text { (beats } / \mathrm{min} \text { ) }\end{array}$ & $1.03(1.004-1.06)$ & .023 & $1.02(1.01-1.04)$ & .007 \\
\hline $\begin{array}{l}\text { Creatinine } \\
(\mathrm{mg} / \mathrm{dL})\end{array}$ & $1.22(0.71-2.12)$ & .47 & $1.66(1.15-2.39)$ & .006 \\
\hline LVEF (\%) & $1.02(0.97-1.07)$ & .43 & $1.0(0.98-1.03)$ & .87 \\
\hline LVESV (mL) & $1.01(0.99-1.04)$ & .24 & $1.01(0.99-1.02)$ & .46 \\
\hline
\end{tabular}

Here only selected results are shown. Additional covariates in the multivariate model without near significant contribution to survival were peripheral vascular disease, chronic obstructive pulmonary disease, pulmonary hypertension, diabetes, coronary artery disease, and sex. Heart rate, LVEF, and LVESV were obtained postprocedurally. $H R$, Hazard ratio; $C I, 95 \%$ confidence interval; $P-P M$, patient-prosthesis mismatch; $L V E F$, left ventricular ejection fraction; $L V E S V$, left ventricular end-systolic volume. and identified expected moderate P-PM to be a risk factor for short- and long-term mortality in patients after AVR. In our study we used measured actual EOA and show that the moderate P-PM has no impact on survival. Pibarot and Dumesnil ${ }^{4}$ suggested an algorithm to avoid P-PM after AVR that relates the expected EOA of the valve prosthesis to the patient's body surface area. After this procedure, neither severe nor moderate P-PM would have been expected in any of our patients. However, this algorithm is not applicable for TAVI because of the unpredictable impact of the native valve and its calcification on postprocedural EOA. Therefore, we measured actual postprocedural EOA in the present study and found the reported incidence for P-PM. Interestingly, preprocedural annulus size had no impact and LVOT diameter only a small impact on the incidence of severe P-PM. In contrast, body surface area greater than $1.885 \mathrm{~m}^{2}$ independently predicted severe P-PM with satisfactory sensitivity (0.71) and specifity (0.70).

The adverse impact of P-PM was more evident in patients with impaired LV function. ${ }^{3}$ In our study, LV function improved immediately after TAVI, which may be due to the lack of an adverse impact of cardiopulmonary bypass and ischemia and reperfusion that occur during conventional AVR. ${ }^{19}$ Yet, a considerable number of our TAVI patients $(27 \%)$ still had a postprocedural LVEF less than $50 \%$, which had a marked effect on 30-month survival. In addition, also within the subgroup of patients with severe P-PM, combination with LVEF less than $50 \%$ presented with a strong trend for decreased 3-month survival $(P=.051)$. Depressed postprocedural LV function could result from more pronounced myocardial remodeling, namely, fibrosis, ${ }^{20}$ or decreased systemic arterial compliance, ${ }^{21}$ which are not relieved by the intervention. Similarly to LVEF, LVESV also influenced the patient's survival. That is to be expected because both parameters are strongly determined by LV systolic function. Although the impact of 
LVEF and LVESV on survival was not confirmed to be independent from other risk factors in the multivariate analysis, we consider this finding noteworthy because these parameters are easily available to the attending physician. In contrast, low LVEDV may indicate diminished myocardial compliance owing to fibrosis, whereas high LVEDV may be due to cardiac dilatation in the course of developing heart failure. Inasmuch as both conditions would be expected to affect survival after TAVI, it is not surprising that no dependence of survival on LVEDV was detected in this study.

\section{CONCLUSIONS}

In conclusion, periprocedural TEE during TAVI allowed identification of risk factors that affect survival in these high-risk patients. Namely, severe P-PM occurred in $7.6 \%$ of patients and was associated with considerably increased early mortality. Combination of this condition with an increased transvalvular peak pressure gradient greater than $10 \mathrm{~mm} \mathrm{Hg}$ even worsened this effect. Although more studies are needed to substantiate such recommendations, this small subgroup of patients might benefit from immediate reintervention, either by redilatation or even by conversion to open cardiac surgery. Finally, it is important to stress that the analysis of echocardiographic data after TAVI and their impact on survival represents only one view of the multitask problem presented by these highrisk patients.

The other members of our TAVI team are Semih Buz, MD, Thorsten Drews, MD, Giuseppe D'Ancona, MD, PhD, Christoph Klein, MD, Katrin Schäfer, Ekatarina Ivanitskaia-Kühn, and Natalia Solowjowa, MD. We thank Anne Gale for editorial assistance.

\section{References}

1. Schueler R, Hammerstingl C, Sinning JM, Nickenig G, Omran H. Prognosis of octogenarians with severe aortic valve stenosis at high risk for cardiovascular surgery. Heart. 2010;96:1831-6.

2. Rahimtoola SH. The problem of valve prosthesis-patient mismatch. Circulation. 1978;58:20-4.

3. Blais C, Dumesnil JG, Baillot R, Simard S, Doyle D, Pibarot P. Impact of valve prosthesis-patient mismatch on short-term mortality after aortic valve replacement. Circulation. 2003;108:983-8.

4. Pibarot P, Dumesnil JG. Hemodynamic and clinical impact of prosthesis-patient mismatch in the aortic valve position and its prevention. J Am Coll Cardiol.2000; 36:1131-41.
5. Cribier A, Eltchaninoff H, Tron C, Bauer F, Agatiello C, Sebagh L, et al. Early experience with percutaneous transcatheter implantation of heart valve prosthesis for the treatment of end-stage inoperable patients with calcific aortic stenosis. J Am Coll Cardiol. 2004;43:698-703.

6. Pasic M. Transapical aortic valve implantation in 175 consecutive patients: excellent outcome in very high-risk patients. J Am Coll Cardiol. 2010;56:813-20.

7. Webb JG, Pasupati S, Humphries K, Thompson C, Altwegg L, Moss R, et al. Percutaneous transarterial aortic valve replacement in selected high-risk patients with aortic stenosis. Circulation. 2007;116:755-63.

8. Pasic M, Buz S, Dreysse S, Drews T, Unbehaun A, Klein C, et al. Transapical aortic valve implantation in 194 patients: problems, complications, and solutions. Ann Thorac Surg. 2010;90:1463-9; discussion 1469-70.

9. Moss RR, Ivens E, Pasupati S, Humphries K, Thompson CR, Munt B, et al. Role of echocardiography in percutaneous aortic valve implantation. J Am Coll Cardiol. 2008;1:15-24.

10. Pasic M, Dreysse S, Drews T, Buz S, Unbehaun A, Kukucka M, et al. Improved technique of transapical aortic valve implantation: "the Berlin addition." Ann Thorac Surg. 2010;89:2058-60.

11. Walther T, Dewey T, Borger MA, Kempfert J, Linke A, Becht R, et al. Transapical aortic valve implantation: step by step. Ann Thorac Surg. 2009;87:276-83.

12. Shanewise JS, Cheung AT, Aronson S, Stewart WJ, Weiss RL, Mark JB, et al ASE/SCA guidelines for performing a comprehensive intraoperative multiplane transesophageal echocardiography examination: recommendations of the American Society of Echocardiography Council for Intraoperative Echocardiography and the Society of Cardiovascular Anesthesiologists Task Force for Certification in Perioperative Transesophageal Echocardiography. J Am Soc Echocardiogr. 1999;12:884-900.

13. Pasic M, Unbehaun A, Dreysse S, Buz S, Drews T, Kukucka M, et al. Transapical aortic valve implantation after previous aortic valve replacement: clinical proof of the "valve-in-valve" concept. J Thorac Cardiovasc Surg. 2011 Aug;142(2): 270-7.

14. Kalavrouziotis D, Rodés-Cabau J, Bagur R, Doyle D, De Larochellière R, Pibarot $\mathrm{P}$, et al. Transcatheter aortic valve implantation in patients with severe aortic stenosis and small aortic annulus. J Am Coll Cardiol. 2011;58: 1016-24.

15. Howell NJ, Keogh BE, Barnet V, Bonser RS, Graham TR, Rooney SJ, et al. Patient-prosthesis mismatch does not affect survival following aortic valve replacement. Eur J Cardiothorac Surg. 2006;30:10-4.

16. Jilaihawi H, Chin D, Spyt T, Jeilan M, Vasa-Nicotera M, Bence J, et al. Prosthesis-patient mismatch after transcatheter aortic valve implantation with the Medtronic-Corevalve bioprosthesis. Eur Heart J. 2010;31:857-64.

17. Rabus MB, Kirali K, Kayalar N, Mataraci I, Yanartas M, Ulusoy-Bozbuga N, et al. Effects of patient-prosthesis mismatch on postoperative early mortality in isolated aortic stenosis. J Heart Valve Dis. 2009;18:18-27.

18. Walther T, Rastan A, Falk V, Lehmann S, Garbade J, Funkat AK, et al. Patient prosthesis mismatch affects short- and long-term outcomes after aortic valve replacement. Eur J Cardiothorac Surg. 2006;30:15-9.

19. Clavel MA, Webb JG, Pibarot P, Altwegg L, Dumont E, Thompson C, et al. Comparison of the hemodynamic performance of percutaneous and surgical bioprostheses for the treatment of severe aortic stenosis. J Am Coll Cardiol. 2009;53: 1883-91.

20. Weidemann F, Herrmann S, Stork S, Niemann M, Frantz S, Lange V, et al. Impact of myocardial fibrosis in patients with symptomatic severe aortic stenosis. Circulation. 2009;120:577-84.

21. Briand M, Dumesnil JG, Kadem L, Tongue AG, Rieu R, Garcia D, et al. Reduced systemic arterial compliance impacts significantly on left ventricular afterload and function in aortic stenosis: implications for diagnosis and treatment. $J \mathrm{Am}$ Coll Cardiol. 2005;46:291-8. 